\title{
A CHARACTERISTICALLY NILPOTENT LIE ALGEBRA CAN BE A DERIVED ALGEBRA
}

\author{
EUGENE M. LUKS ${ }^{1}$
}

\begin{abstract}
An example is constructed of a Lie algebra whose derived algebra has only nilpotent derivations, thus answering a question of Dixmier and Lister.
\end{abstract}

1. Introduction. In a well-known paper in this journal [1], Dixmier and Lister constructed the first example of a characteristically nilpotent Lie algebra, that is, a Lie algebra with only nilpotent derivations. Upon proving that their example has the additional property that it is not the derived algebra of any Lie algebra, they pose the question: If $L$ is any characteristically nilpotent Lie algebra, is it necessarily true that $L$ cannot be a derived algebra? Their proof shows that the answer is yes if every derivation of $L$ maps $L$ into its derived algebra. Leger and Tôgô [2] have shown the answer to be yes under certain other conditions, for example, if every derivation of $L$ annihilates the center of $L$. The purpose of this paper is to resolve Dixmier and Lister's question in the negative. We construct an 18-dimensional Lie algebra, $H$, whose derived algebra, $[H, H]$, is characteristically nilpotent.

2. The example. Let $L$ denote the 16-dimensional Lie algebra over any field $\Phi$, of characteristic not 2 or 5 with basis $\left\{x_{1}, x_{2}, \ldots, x_{16}\right\}$ and multiplication determined by

$$
\begin{aligned}
& {\left[x_{1}, x_{2}\right]=x_{7}, \quad\left[x_{1}, x_{3}\right]=x_{8}, \quad\left[x_{1}, x_{4}\right]=x_{9}, \quad\left[x_{1}, x_{5}\right]=x_{10},} \\
& {\left[x_{1}, x_{6}\right]=x_{13}, \quad\left[x_{1}, x_{7}\right]=x_{15}, \quad\left[x_{1}, x_{8}\right]=x_{16}, \quad\left[x_{2}, x_{3}\right]=x_{11},} \\
& {\left[x_{2}, x_{4}\right]=x_{12}, \quad\left[x_{2}, x_{5}\right]=x_{15}, \quad\left[x_{2}, x_{6}\right]=x_{14}, \quad\left[x_{2}, x_{7}\right]=-x_{16},} \\
& {\left[x_{3}, x_{4}\right]=-x_{13}-(9 / 5) x_{15}, \quad\left[x_{3}, x_{5}\right]=-x_{14}, \quad\left[x_{3}, x_{6}\right]=-x_{16},} \\
& {\left[x_{4}, x_{5}\right]=2 x_{16}, \quad \text { and }\left[x_{i}, x_{j}\right]=0 \text { for } i+j \geqslant 10 .}
\end{aligned}
$$

Note that for distinct $i, j, k$, the products $\left[\left[x_{i}, x_{j}\right], x_{k}\right]$ are all 0 ; thus the Jacobi identity is immediately verified.

REMARK. Recall that an ideal in any Lie algebra, $G$, is called characteristic if it is invariant under all derivations of $G$. Note that, if $I$ and $J$ are characteristic ideals in $G$, then so are $[I, J]$ and the transporter of $I$ to $J$, i.e., $\{x \in G \mid[x, I] \subset J\}$.

Received by the editors February 24, 1975.

AMS (MOS) subject classifications (1970). Primary 17B30, 17B40.

Key words and phrases. Nilpotent Lie algebras, derivations of Lie algebras.

1 The author is pleased to acknowledge research support from Research Corporation. 
Proposition 1. $L$ is characteristically nilpotent.

Proof. For $1 \leqslant i \leqslant 16$, let $I_{i}$ denote the ideal of $L$ spanned by $\left\{x_{j}\right\}_{j \geqslant i}$. The following five statements show that $I_{2}, I_{3}, I_{4}, I_{5}, I_{6}$ are characteristic:

(i) $I_{3}$ is the transporter of $[L, L]\left(=I_{7}\right)$ to 0 .

(ii) $I_{4}$ is the transporter of $L$ to the center of $L\left(=I_{9}\right)$.

(iii) $I_{2}$ is the transporter of $[L, L]$ to $\left[I_{4}, I_{4}\right]\left(=I_{16}\right)$.

(iv) $I_{6}$ is the transporter of $L$ to $\left[I_{2}, I_{2}\right]\left(=I_{11}\right)$.

(v) $I_{5}$ is the transporter of $I_{2}$ to $\left[L, I_{6}\right]\left(=I_{13}\right)$.

Now let $D$ be a derivation of $L$. We shall show $D$ is nilpotent. Since $I_{2}, \ldots, I_{6}$ are characteristic (and $I_{1}=L$ ),

$$
D\left(x_{i}\right) \equiv c_{i} x_{i} \bmod \left(I_{i+1}\right), \quad \text { for } 1 \leqslant i \leqslant 6, \text { where } c_{i} \in \Phi .
$$

Using $\left[x_{1}, x_{2}\right]=x_{7}$ and $\left[x_{1}, I_{3}\right]+\left[I_{2}, x_{2}\right] \subset I_{8}$, we get

$$
D\left(x_{7}\right)=\left[D x_{1}, x_{2}\right]+\left[x_{1}, D x_{2}\right] \equiv\left(c_{1}+c_{2}\right) x_{7} \bmod \left(I_{8}\right) .
$$

Similarly $\left[x_{1}, x_{3}\right]=x_{8}$ and $\left[x_{1}, I_{4}\right]+\left[I_{2}, x_{3}\right] \subset I_{9}$ imply

$$
D\left(x_{8}\right) \equiv\left(c_{1}+c_{3}\right) x_{8} \bmod \left(I_{9}\right) \text {. }
$$

Then

$$
\begin{aligned}
& D\left(x_{16}\right)=D\left(-\left[x_{3}, x_{6}\right]\right)=\left(c_{3}+c_{6}\right) x_{16}, \\
& D\left(x_{16}\right)=D\left(-\left[x_{2}, x_{7}\right]\right)=\left(c_{1}+2 c_{2}\right) x_{16} \\
& D\left(x_{16}\right)=D\left(\left[x_{1}, x_{8}\right]\right)=\left(2 c_{1}+c_{3}\right) x_{16},
\end{aligned}
$$

and

$$
D\left(x_{16}\right)=D\left(1 / 2\left[x_{4}, x_{5}\right]\right)=\left(c_{4}+c_{5}\right) x_{16},
$$

so $c_{3}+c_{6}=c_{1}+2 c_{2}=2 c_{1}+c_{3}=c_{4}+c_{5}$. Next

$$
D\left(x_{15}\right)=D\left(\left[x_{1}, x_{7}\right]\right) \equiv\left(2 c_{1}+c_{2}\right) x_{15} \bmod \left(I_{16}\right) \text {. }
$$

In particular, this last relation implies that $D\left(x_{15}\right)$ has no $x_{14}$ component, which we use, noting $\left[I_{3}, x_{5}\right]+\left[x_{2}, I_{6}\right] \subset\left(x_{14}, x_{16}\right)$, to get

$$
D\left(x_{15}\right)=D\left(\left[x_{2}, x_{5}\right]\right) \equiv\left(c_{2}+c_{5}\right) x_{15} \bmod \left(I_{16}\right) .
$$

Thus $2 c_{1}+c_{2}=c_{2}+c_{5}$. Also,

$$
D\left(x_{14}\right)=D\left(\left[x_{2}, x_{6}\right]\right) \equiv\left(c_{2}+c_{6}\right) x_{14} \bmod \left(I_{15}\right),
$$

and

$$
D\left(x_{14}\right)=D\left(-\left[x_{3}, x_{5}\right]\right) \equiv\left(c_{3}+c_{5}\right) x_{14} \bmod \left(I_{15}\right),
$$

so $c_{2}+c_{6}=c_{3}+c_{5}$. Finally,

$$
D\left(x_{13}\right)=D\left(\left[x_{1}, x_{6}\right]\right) \equiv\left(c_{1}+c_{6}\right) x_{13} \bmod \left(I_{14}\right)
$$

and 


$$
D\left(x_{13}\right)=D\left(-\left[x_{3}, x_{4}\right]-(9 / 5) x_{15}\right) \equiv\left(c_{3}+c_{4}\right) x_{13} \bmod \left(I_{14}\right),
$$

whence $c_{1}+c_{6}=c_{3}+c_{4}$. The above relations on the $c_{i}$ yield $c_{1}=c_{2}=\cdots$ $=c_{6}=0$, i.e., $D\left(x_{i}\right) \subset I_{i+1}$ for $i=1,2, \ldots, 6$. Hence $D^{6}(L) \subset[L, L]$. Since $L$ is nilpotent, $D$ is a nilpotent derivation.

REMARK. An analogous proof shows that every automorphism of $L$ is unipotent.

Proposition 2. $L$ is a derived algebra.

Proof. Let $D_{1}$ denote the derivation of $L$ such that

$$
\begin{aligned}
& D_{1}\left(x_{3}\right)=x_{7}, D_{1}\left(x_{4}\right)=2 x_{8}, D_{1}\left(x_{5}\right)=3 x_{9}+2 x_{11}, \\
& D_{1}\left(x_{6}\right)=4 x_{10}+5 x_{12}, \quad D_{1}\left(x_{8}\right)=x_{15}, \quad D_{1}\left(x_{9}\right)=2 x_{16}, \\
& D_{1}\left(x_{11}\right)=-x_{16}, \quad \text { with } D_{1}\left(x_{i}\right)=0 \text { otherwise } .
\end{aligned}
$$

Let $D_{2}$ denote the derivation of $L$ such that

$$
\begin{aligned}
& D_{2}\left(x_{1}\right)=x_{2}, D_{2}\left(x_{2}\right)=x_{3}, D_{2}\left(x_{3}\right)=x_{4}, D_{2}\left(x_{4}\right)=x_{5}, \\
& D_{2}\left(x_{5}\right)=x_{6}, D_{2}\left(x_{6}\right)=0, D_{2}\left(x_{7}\right)=x_{8}, D_{2}\left(x_{8}\right)=x_{9}+x_{11}, \\
& D_{2}\left(x_{9}\right)=x_{10}+x_{12}, D_{2}\left(x_{10}\right)=x_{13}+x_{15}, D_{2}\left(x_{11}\right)=x_{12}, \\
& D_{2}\left(x_{12}\right)=-x_{13}-(4 / 5) x_{15}, D_{2}\left(x_{13}\right)=x_{14}, D_{2}\left(x_{14}\right)=-x_{16}, \\
& D_{2}\left(x_{15}\right)=0, D_{2}\left(x_{16}\right)=0 .
\end{aligned}
$$

One finds that $\left[D_{1}, D_{2}\right]=$ ad $\left(x_{1}\right)$. Hence we may extend $L$ to a Lie algebra $H=\left(\tilde{D}_{1}, \tilde{D}_{2}\right)+L$ in which $\left[\tilde{D}_{i}, x_{j}\right]=D_{i}\left(x_{j}\right),\left[\tilde{D}_{1}, \tilde{D}_{2}\right]=x_{1}$ and products in $L$ are as before. Note that $[H, H]=L$.

\section{REFERENCES}

1. J. Dixmier and W. G. Lister, Derivations of nilpotent Lie algebras, Proc. Amer. Math. Soc. 8 (1957), 155-158. MR 18, 659.

2. G. Leger and S. Tôgô, Characteristically nilpotent Lie algebras, Duke. Math. J. 26 (1959), 623-628. MR 22 \#5659.

Department of Mathematics, Bucknell University, Lewisburg, Pennsylvania 17837 